\title{
Inhibition of Aurora-B kinase activity confers antitumor efficacy in preclinical mouse models of early and advanced gastrointestinal neoplasia
}

\author{
DENIS G. ALFEREZ ${ }^{1,2}$, ROBERT A. GOODLAD ${ }^{1,5}$, RAJESH ODEDRA ${ }^{2}$, PATRIZIA SINI ${ }^{2}$, \\ CLAIRE CRAFTER $^{2}$, ANDERSON J. RYAN ${ }^{2,3}$, STEPHEN R. WEDGE ${ }^{2}$, NICHOLAS A. WRIGHT $^{1,4}$, \\ ELIZABETH ANDERSON ${ }^{2}$ and ROBERT W. WILKINSON ${ }^{2,6}$
}

\begin{abstract}
${ }^{1}$ Histopathology Unit, London Research Institute, Cancer Research UK, Lincoln's Inn Fields, London; ${ }^{2}$ Oncology iMED, AstraZeneca Pharmaceuticals, Alderley Park, Macclesfield, Cheshire; ${ }^{3}$ Gray Institute for Radiation Oncology and Biology, University of Oxford, Roosevelt Drive, Headington, Oxford; ${ }^{4}$ Centre for Digestive Diseases, Blizard Institute, Blizard Building, Newark Street, Whitechapel, London; ${ }^{5}$ Imperial College, Hammersmith Hospital, London, UK
\end{abstract}

Received April 20, 2012; Accepted June 4, 2012

DOI: $10.3892 /$ ijo.2012.1580

\begin{abstract}
The Aurora family of kinases, play a fundamental role in cell division and are overexpressed in several cancers including colon. The activity of barasertib-hQPA, a selective inhibitor of Aurora-B kinase (ABK) was investigated in a range of preclinical models of gastrointestinal cancer. Treatment with barasertib-hQPA produced anti-proliferative and cytotoxic effects across a panel of human colorectal cancer (CRC) cell lines in vitro. Prodrug, barasertib [48-h subcutaneous (s.c.) infusion; $150 \mathrm{mg} / \mathrm{kg} /$ day] inhibited the growth of SW620, Colo205, HCT116 human colorectal tumor xenografts in nude mice significantly (Student's t-test, $\mathrm{P}<0.05, \mathrm{n}=10-12$ per group). Flow cytometric analysis of single cells from disaggregated barasertib-treated SW620 tumors revealed a decrease in phosphorylated histone $\mathrm{H} 3(\mathrm{phH} 3)$ and an increase in tumor cells with $\geq 4 \mathrm{~N}$ DNA content $\mathrm{P}<0.05$ ). The activity of barasertib was then examined in $A p c^{\mathrm{Min} /+}$ mice, a spontaneous model of early intestinal neoplasia. Macroscopic evaluation of the small intestine revealed that barasertib treatment $[25 \mathrm{mg} / \mathrm{kg}$ intra-peritoneal (i.p.) Q1Dx4 each week for 3 weeks] of 8-week old $A p c^{\mathrm{Min} /+}$ mice produced a $39 \%$ reduction in macroadenoma
\end{abstract}

Correspondence to: Dr Robert W. Wilkinson, ${ }^{6}$ Present address: Oncology Research, MedImmune Limited, Aaron Klug Building, Granta Park, Cambridge, CB21 6GH, UK

E-mail: wilkinsonr@MedImmune.com

Abbreviations: CRC, colorectal cancer; APC, adenomatous polyposis coli; Min, Multiple intestinal neoplasia $\mathrm{Apc} \mathrm{Min} /+^{\mathrm{M}}$ ABK, Aurora-B kinase; phH3, phospho-histone H3; Barasertib-hQPA, barasertibhydroxy quinazoline pyrazol anilide

Key words: colorectal cancer, neoplasia, early disease, Aurora-B kinase inhibitors, barasertib, barasertib-hQPA number $(\mathrm{P}=0.02)$ and a $43 \%$ reduction in overall adenoma burden $(\mathrm{P}=0.02)$ compared with vehicle-treated controls. Quantification of microscopic adenomas revealed a $>64 \%$ reduction in the number of adenomas spanning more than one villus. Histological analysis of these adenomas revealed a number of distinct changes in barasertib-treated $A p c^{\mathrm{Min} /+}$ mice, including a $94 \%$ reduction in the proportion of phosphohistone $\mathrm{H} 3$-positive cells $(\mathrm{P}<0.001)$ and a 53\% reduction in the number of cells per adenoma $(\mathrm{P}=0.001)$. These results provide a scientific rationale for investigating $\mathrm{ABK}$ inhibitors as a treatment for intestinal cancer.

\section{Introduction}

Colorectal cancer (CRC) is the second most common cause of cancer-related death in the Western world. In the US, CRC is the third most common cancer with nearly 150,000 new cases and 50,000 deaths estimated in 2009 (1). After surgery, the mainstay of systemic therapy is a cytotoxic regimen based on 5-fluorouracil combined with folinic acid, oxaliplatin and/ or irinotecan. To this, targeted agents have been added such as the cetuximab or bevacizumab monoclonal antibodies directed against epidermal growth factor receptor (EGFR) or vascular endothelial growth factor (VEGF), respectively. Despite improvements in cytotoxic chemotherapy and the introduction of novel agents, up to $50 \%$ of patients undergoing apparently curative surgery relapse and die of metastatic disease. Moreover, a substantial proportion of CRC patients $(\sim 20 \%)$ present with metastatic disease for whom systemic chemotherapy is palliative at best (2). Thus there is a sustained need for new antitumor therapies that can be used either alone or in combination with existing treatments.

The Aurora kinases are a family of three serine/threonine kinases (Aurora A, B and C) that are important for the accurate segregation of chromosomes into daughter cells during mitosis (3). Aurora-A kinase (AAK) localizes to the centrosomes and spindle poles where it is required for correct spindle assembly. 
In contrast, Aurora-B kinase (ABK) is a chromosome passenger protein required for phosphorylation of histone $\mathrm{H} 3$ (phH3), correct alignment of chromosomes on the metaphase plate and cytokinesis. Expression of the third family member, Aurora-C kinase, is mostly restricted to the testis where it may play a role in meiosis (4). Expression of the Aurora kinases is frequently elevated in human tumors including those of the colon and rectum (5). In particular, increased ABK mRNA levels have been shown recently to be associated with poor overall survival (OS) in patients with metastatic CRC (6).

In CRC, one of the earliest molecular events is loss of function of the adenomatous polyposis coli (APC) gene product. Somatic mutations in the APC gene occur in the majority of sporadic CRC (7). Germline APC mutations are found in the autosomal dominant condition, familial adenomatous polyposis (FAP), where patients develop numerous colorectal adenomas and early onset CRC (8). APC plays a central role in the regulation of $\beta$-catenin, a key intracellular mediator of the Wnt signaling pathway. In addition to its critical role in the Wnt signaling pathway, the product of the APC gene has been shown to be involved in multiple cellular functions including chromosome segregation $(9,10)$. Recent studies, using CRC cell lines and patient samples, have shown mutated APC upregulates expression of the anti-apoptotic protein survivin (11) which in turn activates ABK leading to catalysis of mitosis (12). This may explain the increase in mitotic figures and cellular proliferation that is one of the hallmarks of CRC pathology.

Targeting kinases, such as ABK, that are involved in mitosis is an attractive therapeutic strategy, with the potential to provide similar efficacy to conventional cytotoxic agents but with fewer side effects, such as peripheral neuropathy. Barasertib (formerly AZD1152) is a dihydrogen phosphate prodrug which is rapidly converted to the hydroxyquinazoline pyrazol anilide (barasertib-hQPA) in plasma. BarasertibhQPA is a highly potent and selective inhibitor of $\mathrm{ABK}$ $(\mathrm{Ki}=0.36 \mathrm{nmol} / \mathrm{l})$ compared to AAK $(\mathrm{Ki}=1369 \mathrm{nmol} / \mathrm{l})$. Consistent with what is known about the function of ABK, treatment of tumor cells with barasertib hQPA either in vitro or in vivo induces chromosome misalignment and failure of cytokinesis resulting in polyploidy and cell death (13).

Most previous studies on the effects of barasertib on solid tumor growth have been carried out in heterotopic tumor xenograft models where CRC cell lines have been grown subcutaneously and not within the context of the GI tract microenvironment. An alternate model that enables GI lesions that develop in situ to be examined, is the $A p c^{\mathrm{Min} /+}$ model of spontaneous intestinal neoplasia. $A p c^{\mathrm{Min} /+}$ mice carry a germ line mutation in the APC gene comparable to the mutation identified in patients with inherited FAP (14). Like patients with FAP, $A p c^{\mathrm{Min} /+}$ mice develop multiple adenomas throughout the intestine which progress to invasive tumors with a frequency that depends on genetic background (15). Allowing for a close association between the $A p c^{\mathrm{Min} /+}$ mouse model and human CRC $(14,16,17)$.

In the present report, we describe the antitumor results of more extensive experiments evaluating barasertib activity in a panel of human CRC cell lines grown in vitro and as xenografts in immunocompromised nude mice; and in the autochthonous $A p c^{\mathrm{Min} /+}$ mouse model. We confirm our preliminary observa- tions and show that barasertib-hQPA is a potent inhibitor of CRC cell line growth both in vitro and in vivo. As expected, growth inhibition is accompanied by reduced phospho-histone $\mathrm{H} 3$ (phH3) levels, increased numbers of polyploid cells and an increase in apoptotic cells. In the $A p c^{\mathrm{Min} /+}$ model, treatment with barasertib significantly reduced adenoma burden in the small bowel.

\section{Materials and methods}

Reagents. Chemicals were purchased from Sigma (Poole, UK), unless otherwise stated. Barasertib-hQPA is an acetanilide substituted pyrazole aminoquinazoline and barasertib is a dihydrogen phosphate prodrug of barasertib-hQPA (18). Both were synthesized by AstraZeneca Pharmaceuticals, Alderley Park, Macclesfield, Cheshire, UK.

Animals. All procedures, including breeding and pharmacology studies using $A p c^{\mathrm{Min} /+}$ mice (originally obtained from Amy R. Moser, University of Wisconsin, Madison, WI, USA) and Swiss nude mice ( $n u / n u$ genotype; AstraZeneca, UK), were approved by the Cancer Research UK and AstraZeneca animal ethics committees, respectively and carried out in accordance with the UK Home Office Animals (Scientific Procedures) Act, 1986.

Cell lines. The DMS114, HCT116, RKO, SW620, Colo205, WiDr, HCT8, Colo320DM, and SW116 CRC cell lines were obtained from the European Collection of Animal Cell Cultures (ECACC) or American Type Culture Collection (ATCC). Cells were cultured in RPMI-1640 medium (Sigma) supplemented with $10 \%$ fetal calf serum and $2 \mathrm{mmol} / 1$ L-glutamine and maintained at $37^{\circ} \mathrm{C}$ in a humidified atmosphere of $5 \% \mathrm{CO}_{2} / 95 \%$ air as described previously (13).

Proliferation assay. Cells in log-phase growth were seeded in 96-well tissue culture plates and allowed to adhere overnight. Cells were then exposed to increasing concentrations of barasertib-hQPA ranging from 0.003 to $3 \mu \mathrm{mol} / 1$. After incubation for $96 \mathrm{~h}$, cells were fixed in $2 \%$ formaldehyde for $30 \mathrm{~min}$, washed in PBS and stained with Hoechst dye to visualize the nuclei. Replicate plates were also stained at the start of dosing to provide a day zero reading. Cells were then imaged on the ArrayScan II platform (Cellomics, Pittsburgh, PA, USA) to determine cell number. Individual $\mathrm{GI}_{50}$ values were calculated using the cell count readings at time zero and at the end of the treatment period and the data were summarized using the geometric mean (the average of the logarithmic values converted back to a base 10 number, $n=2-3$ ).

Apoptosis assay. The apoptotic effect was determined by measuring the active form of BAK protein, an early marker of apoptosis. Cells in 96-well plates were exposed to increasing concentrations of barasertib-hQPA for $24 \mathrm{~h}$. After treatment, the compound was washed out and the cells left to recover for 48, 96, 120 or $144 \mathrm{~h}$. Cells were then fixed and stained with Hoechst dye (1:10,000, Molecular Probes, Eugene, CA, USA) to assess cell number and with anti-Bak antibody (Y164)(1:1000, Abcam, Cambridge, UK) that recognizes the active form of BAK to determine the number of apoptotic cells. Images, from 
3-4 replicate wells, were taken and endpoints quantified using the Cellomics Arrayscan II platform (Cellomics) as described previously (19).

Human tumor xenografts. Human tumor xenografts were established in 8- to 12-week old female nude mice by injecting $0.1 \mathrm{ml}$ of a suspension of $3 \times 10^{5}$ cells (either SW620, Colo205 or HCT116) mixed 50:50 with Matrigel (BD Biosciences, $\mathrm{UK})$ into the dorsal flank. Once tumors were established $\left(0.2-0.3 \mathrm{~cm}^{3}\right)$ animals were randomized into treatment groups ( $\mathrm{n}=8$-11 per group); barasertib prepared in Tris buffer $\mathrm{pH} 9.0$ or vehicle alone was administered either as a continuous 48-h infusion via subcutaneous (s.c.) implanted osmotic mini-pumps (Model 2001D, Durect Corp, USA: two $24 \mathrm{~h}$ pumps implanted sequentially) in accordance with the manufacturer's instructions or, in SW620, as an intra-peritoneal (i.p.) injection daily for 4 consecutive days (Q1Dx4). Tumors were measured up to three times per week with calipers, tumor volumes were calculated and the data plotted using the geometric mean for each group versus time. Tumor volume and percentage tumor growth inhibition were calculated as described previously (20).

For pharmacodynamic (PD) studies, mice (3 per group) were humanely culled at the end of barasertib administration and tumor xenografts harvested and snap frozen. Cell suspensions were prepared from the frozen tumors using a tissue disaggregation system (Medimachine; BD Biosystems, Oxford, UK). Estimation of cellular DNA content and phH3 expression and was undertaken using previously described immunostain and flow cytometry protocols $(13,21)$. In brief, tumor cells were stained using propidium iodide (Sigma), a rabbit polyclonal IgG anti-phH3 (Upstate Biotechnology, Millipore, Abingdon, UK) and a fluorescein-conjugated goat anti-rabbit IgG (Jackson ImmunoResearch, Stratech, Suffolk, UK). Samples were run on a FACSCalibur flow cytometer and assessed using CellQuest software (both Becton-Dickinson, Oxford, UK).

In vivo adenoma model. Eight-week old $A p c^{\mathrm{Min} /+}$ mice were randomized into groups of 15 animals and treated with either vehicle (see above) or barasertib (25 mg/kg) Q1Dx4 each week for 3 consecutive weeks. Samples were taken $2 \mathrm{~h}$ after the last dose of barasertib or vehicle. To assess proliferation, bromodeoxyuridine (BrdU, $50 \mathrm{mg} / \mathrm{kg}$ i.p.) was given $1 \mathrm{~h}$ before mice were humanely culled.

Macroscopic assessment of tumor burden. At necropsy, spleens were removed and weighed as a marker of tumor load. The small bowel SB (duodenum, jejunum, and ileum), and colon were removed and cleaned by rinsing with cold PBS). The SB was divided into three equal sections: proximal (SB1), middle (SB2) and distal (SB3). These sections and the colon were dissected longitudinally using a recently described cutting guide (22) and spread onto filter paper before being fixed in Carnoy's fluid for $1 \mathrm{~h}$ and stored in $70 \%$ ethanol until analysis. Macroscopic adenoma quantification was carried out using a dissecting microscope as described previously (23) to assess the number, size and overall tumor burden.

Microscopic adenoma assessment. After measurement of adenoma number and diameter, fixed distal SB3 tissue from six mice from each group were rolled and processed to paraffin blocks in a standard manner. Eight $4 \mu \mathrm{m}$ step sections, taken at $200 \mu \mathrm{m}$ intervals, were stained with haematoxylin and eosin (H\&E) and scored morphologically. Microadenomas were assigned to a category from 1-4: adenomas that remained within the limits of a single villus were classified as category 1 ; those within the space of $>1-5$ villi were classified as category 2 ; $>5-10$ villi as category 3 , and $>10$ villi as category 4 (24).

Immunohistochemistry (IHC). Sections of formalin-fixed paraffin-embedded tissue specimens (distal small bowel) from vehicle and barasertib-treated mice were stained for BrdU uptake using sequential incubation with a mouse antiBrdU monoclonal primary antibody (Abcam), corresponding biotin-conjugated rabbit anti-mouse secondary antibody and developed with streptavidin-peroxidase reagent and DAB as previously described (24). Proliferation activity was quantified using thirty well-oriented crypts per animal ( $n=6$ per group) also as described previously $(23,25)$.

Expression of phH3 was determined in sections cut from specimens of the distal small intestine, using sequential incubation with a mouse anti-pHH3-(Ser10) monoclonal primary antibody (Sigma, St. Louis, MO, USA), a biotinconjugated rabbit anti-mouse polyclonal secondary antibody (DakoCytomation Ltd., Ely, UK), and a streptavidin-peroxidase reagent that was developed in DAB followed by counterstaining with haematoxylin. All cells within 25 hemi-crypts per animal in the distal intestine were examined at high magnification (x40 objective) and DAB positive vs. haematoxylin counterstained cells were counted (25). Six animals per group were assessed.

Statistical analysis. All IHC measurements were scored with the slide reader blinded to the treatment. Results are presented as group mean \pm standard error of the mean (SEM). One-way analysis of variance (ANOVA) and Student's t-tests were carried out to test for statistical significance of any effects in barasertib-treated group versus control (P-value of $\leq 0.05$ was considered to be statistically significant). All statistics were performed using Minitab Statistical Software, Release 10.5 Xtra (Minitab Ltd., Coventry, UK) and Graphpad Prism version 4.0b, 2004.

\section{Results}

Effect of barasertib-hQPA on cell growth and survival in vitro. It has been shown previously that barasertib-hQPA is a potent and selective inhibitor of $\mathrm{ABK}$ which inhibits histone $\mathrm{H} 3$ phosphorylation and induces polyploidy in CRC cell lines (HCT116 and SW620) (13,26). Here, we investigated the anti-proliferative and cytotoxic effects of barasertib-hQPA in a panel of CRC cell lines in vitro, exposed to increasing concentrations of barasertib-hQPA for $96 \mathrm{~h}$. BarasertibhQPA treatment potently reduced the proliferation of tumor cells as determined by cell number. In a panel of 9 tumor cell lines, the concentrations of drug that reduced cell growth by $50 \%$ were $<0.015 \mu \mathrm{M}$ in 5 of the lines and between 0.015 $2.235 \mu \mathrm{M}$ in the remainder (Table I). To determine whether barasertib-hQPA could also induce apoptosis, a BAK assay was performed on four of the lines which had shown greatest sensitivity in the proliferation assay. Exposure to barasertib- 
Table I. Barasertib-hQPA inhibits the proliferation of a panel of colon tumor cell lines.

\begin{tabular}{lc}
\hline Cell line & Proliferation $\mathrm{GI}_{50}, \mu \mathrm{M}$ \\
\hline DMS114 & $0.003 \pm 0.001$ \\
HCT116 & $0.010 \pm 0.002$ \\
RKO & $0.012 \pm 0.008$ \\
SW620 & $0.013 \pm 0.002$ \\
Colo205 & $0.014 \pm 0.008$ \\
WiDr & $0.109 \pm 0.006$ \\
HCT8 & $0.503 \pm 0.076$ \\
Colo320DM & $1.075 \pm 0.508$ \\
SW116 & $2.235 \pm 0.658$ \\
\hline
\end{tabular}

Cells were seeded in 96-well plates and exposed to a range of doses of barasertib-hQPA for $96 \mathrm{~h}$ and then fixed and stained with Hoechst to allow the assessment of cell number on the ArrayScan II. Data were summarized using the geometric mean \pm SEM $(n=2-3)$.

hQPA for $24 \mathrm{~h}$, followed by washout for up to $144 \mathrm{~h}$ resulted in significant inhibition of cell growth, at drug concentrations $\geq 0.01 \mu \mathrm{M}$ (Fig. 1A-D). This was accompanied by increased BAK protein (Fig. 1E-H). Both the washout time required for tumor cells to become apoptotic and the extent of apoptosis varied across the cell lines tested. The induction of apoptosis was particularly marked in the Colo-205 and RKO cell lines (Fig. 1E and H, respectively) where $40-60 \%$ of cells stained positive for BAK at drug concentrations $\geq 0.1 \mu \mathrm{M}$.

Barasertib treatment leads to antitumor and pharmacodynamic effects in a range of human CRC xenografts. Pharmacokinetic studies in rodents have previously indicated that barasertib is rapidly converted in vivo to the active moiety barasertib-hQPA (18). To further evaluate the antitumor potential in gastrointestinal malignancies, barasertib $(150 \mathrm{mg} / \mathrm{kg} / \mathrm{day}$; s.c. minipump infusion over $48 \mathrm{~h}$ ) was tested in nude mice subcutaneously implanted with CRC human tumor xenografts. In SW620, HCT116 and Colo205 xenografts significant tumor growth inhibitions of $79 \%(\mathrm{P}<0.001$, day 23$), 60 \%(\mathrm{P}<0.001$, day 25) and $81 \%(\mathrm{P}<0.05$, day 21) were observed, respectively (Fig. 2A-C). Colo205 xenografts appeared the most sensitive to treatment with a mean tumor volume $( \pm$ SEM) on day 21 after cell implantation, of $0.42 \pm 0.19 \mathrm{~cm}^{3}$ for the barasertib group compared to $2.24 \pm 0.75 \mathrm{~cm}^{3}(\mathrm{P}<0.05)$ for the vehicle control animals (Fig. 2C). A significant antitumor response was also observed in SW620 xenografts when barasertib was administered using a well-tolerated once-daily intraperitoneal (i.p) schedule $(25 \mathrm{mg} / \mathrm{kg}$; i.p. Q1Dx4): the mean tumor volume $( \pm$ SEM) on day 24 after cell implantation for barasertib-treated mice was $0.74 \pm 0.07 \mathrm{~cm}^{3}$, compared to vehicle control treated mice, which had a mean tumor volume of $1.26 \pm 0.13 \mathrm{~cm}^{3}(\mathrm{P}<0.05)$ (Fig. 2D).

To confirm that barasertib inhibited $\mathrm{ABK}$ activity in vivo the CRC human tumor xenografts were analyzed $2 \mathrm{~h}$ after termination for biomarker effects including phH3 expression, a proximal downstream marker of ABK signaling (27) and DNA ploidy. The barasertib infusion schedule $(150 \mathrm{mg} / \mathrm{kg} / 48 \mathrm{~h})$ led to a significant reduction in the proportion of $\mathrm{phH} 3$ positive tumor cells in all three CRC xenografts (SW620, HCT116 and Colo205) when compared to respective controls (Fig. 2E). Flow cytometric analysis of the DNA content of SW620 and HCT116 tumors also revealed an increased proportion of cells with a $4 \mathrm{~N}$ and $>4 \mathrm{~N}$ DNA content from animals that received barasertib versus vehicle control treated mice (Fig. 2F and G). This accumulation of cells with a $4 \mathrm{~N}$ and $>4 \mathrm{~N}$ DNA content is consistent with failed cytokinesis and continued cell cycle progression and endoreduplication following inhibition of ABK activity. Such a trend of increased DNA content was not as clear in the Colo205 xenografts receiving barasertib, perhaps related to their propensity to undergo apoptosis. A similar pharmacodynamic trend (i.e. reduction in tumor $\mathrm{PhH} 3$ but increase in $4 \mathrm{~N}$ and $>4 \mathrm{~N}$ cells treated with barasertib versus control) was also observed in the SW620 xenograft model following daily intraperitoneal administration of barasertib ( $25 \mathrm{mg} / \mathrm{kg} /$ day) for 4 days (data not shown).

Barasertib significantly reduces adenoma burden in Apc ${ }^{\mathrm{Min} /+}$ mice. To investigate the efficacy of inhibiting ABK activity over time in the $A p c^{\mathrm{Min} /+}$ model we adapted the i.p. barasertib schedule previously shown to be efficacious in the SW620 tumor xenograft model. $A p c^{\mathrm{Min} /+}$ mice were treated for three consecutive weeks with either barasertib $(25 \mathrm{mg} / \mathrm{kg} /$ day i.p. Q1Dx4 weekly, for 3 cycles) or vehicle alone, and at the end of the treatment phase, intestinal tissue was examined for adenoma formation/development. Barasertib, compared with vehicletreated mice, reduced adenoma number in each of the small bowel (SB) segments SB1, 2 and 3 (data not shown). When the whole SB was considered (SB All) barasertib reduced the mean number of adenomas by $39 \%$ from $52.31 \pm 6.18$ to $31.87 \pm 5.64$ $(\mathrm{P}<0.05)$; Table II. There were much fewer adenomas in the colon than in the SB, due to the lower incidence of adenomas occurring in the colon in this model (22). Although a similar reduction in the number of adenomas in the colon (38\%) was seen in barasertib versus vehicle-treated mice, this did not reach statistical significance (Table II).

When compared with vehicle-treated controls, the mean adenoma diameter in barasertib-treated mice appeared smaller in both the SB and colon, although in neither case was this statistically significant. Adenoma burden (a measure derived from tumor number and diameter) in the small bowel was significantly reduced by $43 \%$ from $22.09 \pm 3.14$ to $12.67 \pm 2.13 \mathrm{~mm}^{3}(\mathrm{P}<0.05)$ (Table II). Whilst there was also a reduction in adenoma burden with barasertib in the colon versus vehicle-treated $A p c^{\mathrm{Min} /+}$ mice of $55 \%$ this was again not statistically significant.

In addition to analyzing the effects of barasertib on macroscopic adenoma formation and growth, a survey of microscopic adenomas was undertaken in which tumors were categorized according to size: category 1 represented an adenoma remaining within the limits of a single villus; category 2, adenomas within the space of $>1-5$ villi; category 3 , adenoma size $>5-10$ villi; category 4 , adenomas $>10$ villi in size. In barasertib-treated animals there were significantly fewer larger microadenomas (by $64,68,67 \%$ in categories $2,3,4$, respectively) suggesting that there are significant effects of barasertib on all, but the very smallest adenomas (Table III). 
A

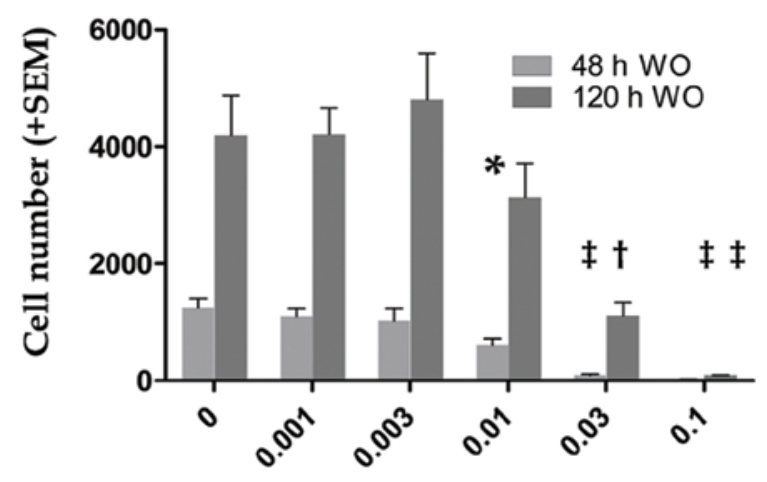

B

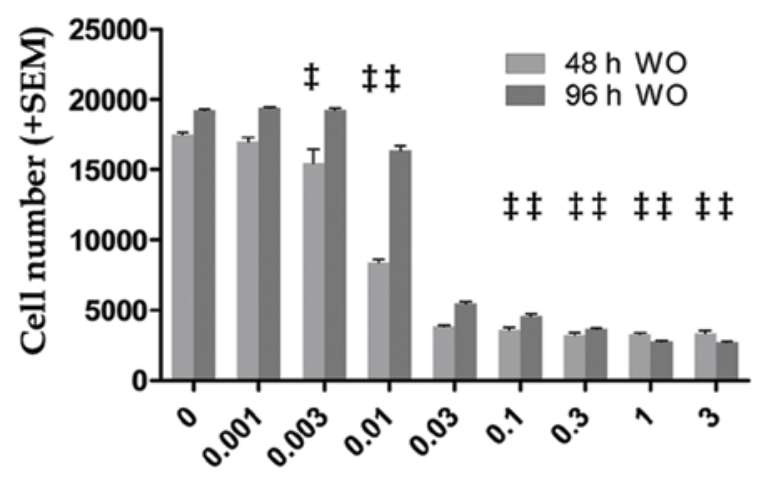

C

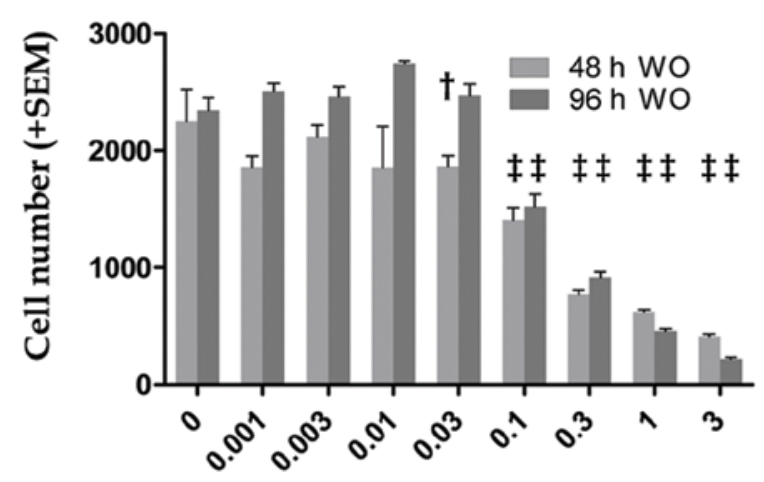

E

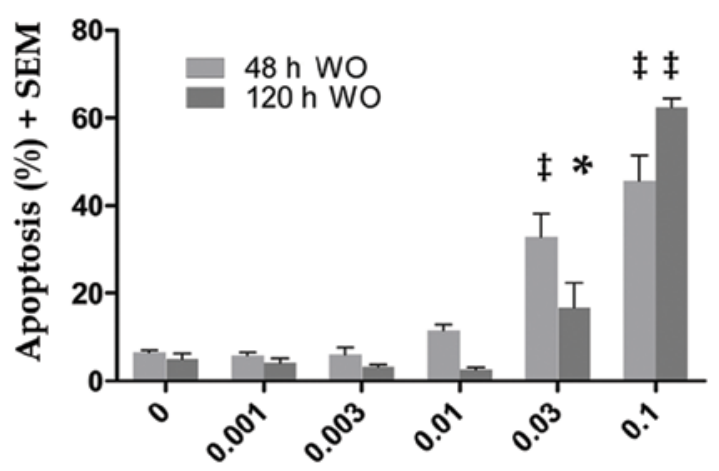

$\mathbf{F}$

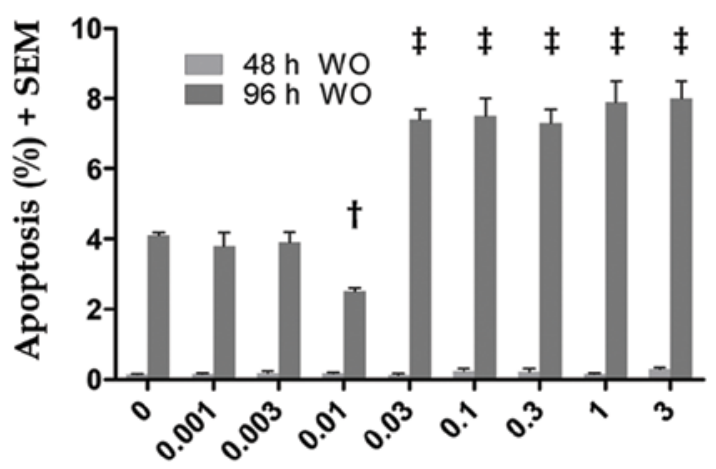

G

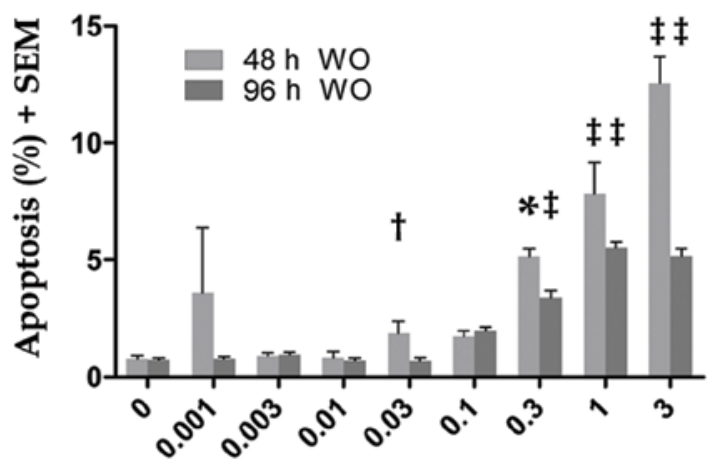

D

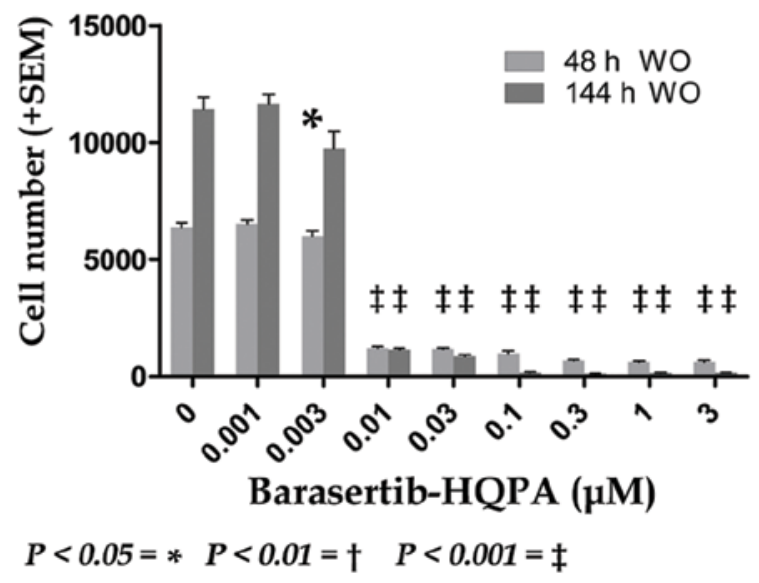

$\mathbf{H}$

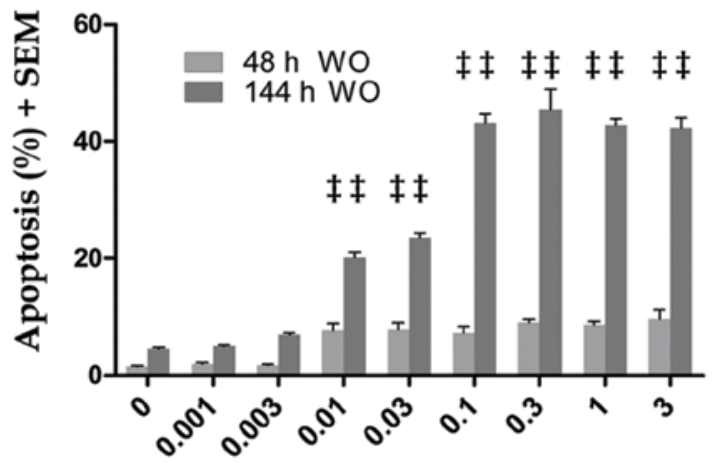

Barasertib-HQPA $(\mu \mathrm{M})$

Figure 1. Anti-proliferative and apoptotic effect of barasertib-hQPA in a panel of CRC cell lines. CRC cells were seeded in 96-well plates and exposed to a range of doses of barasertib-hQPA for $24 \mathrm{~h}$ [Colo205 (A and E), HCT-116 (B and F), SW620 (C and G) and RKO (D and H)]. The compound was washed out (WO) and the cells left to recover over various time points 48, 96, 120 and $144 \mathrm{~h}$. Cells were then stained for BAK protein and nuclei (Hoechst). Cell number (A-D) and apoptosis (E-H) were assessed using the Cellomics ArrayScan II platform. Data represent the mean \pm SEM. Statistical analysis was carried out using standard Dunnett's multiple comparisons tests, ${ }^{*} \mathrm{p}<0.05,{ }^{\dagger} \mathrm{p} \leq 0.01,{ }^{\dagger} \mathrm{p} \leq 0.001$, where applicable. 
A

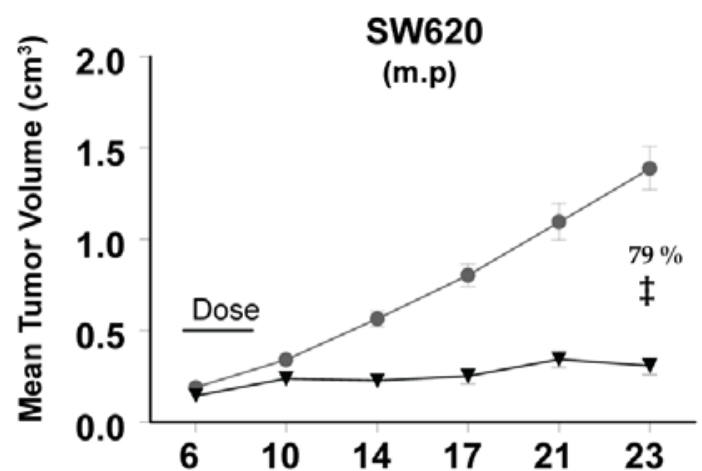

B

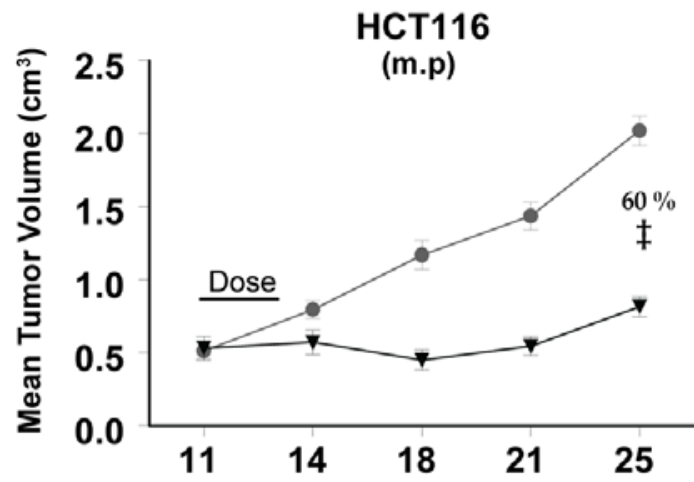

C

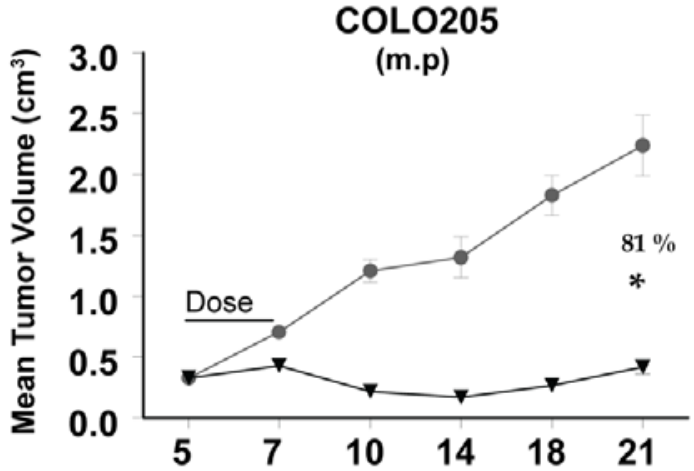

D

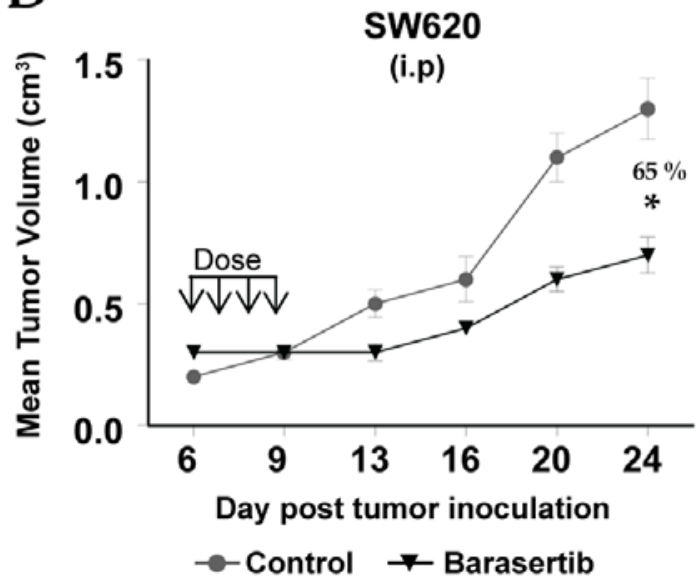

E

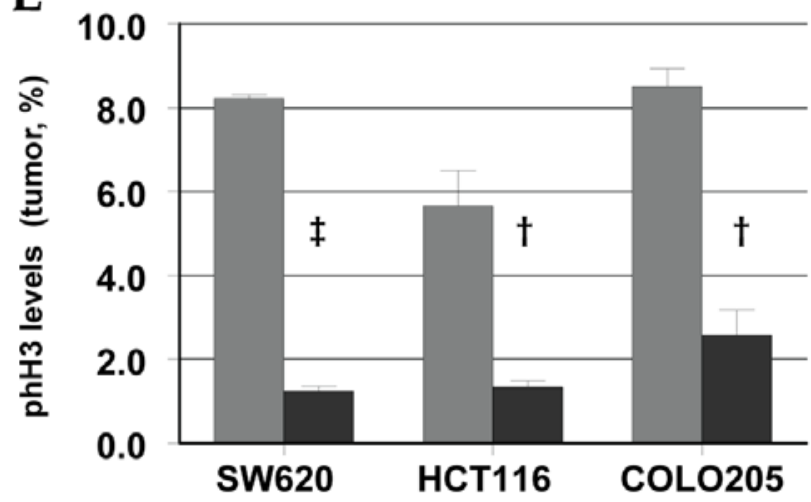

$\mathbf{F}$

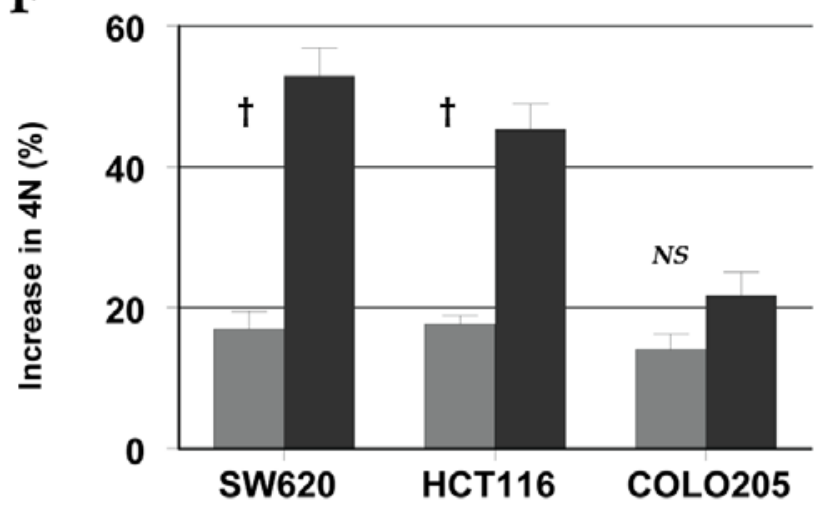

G

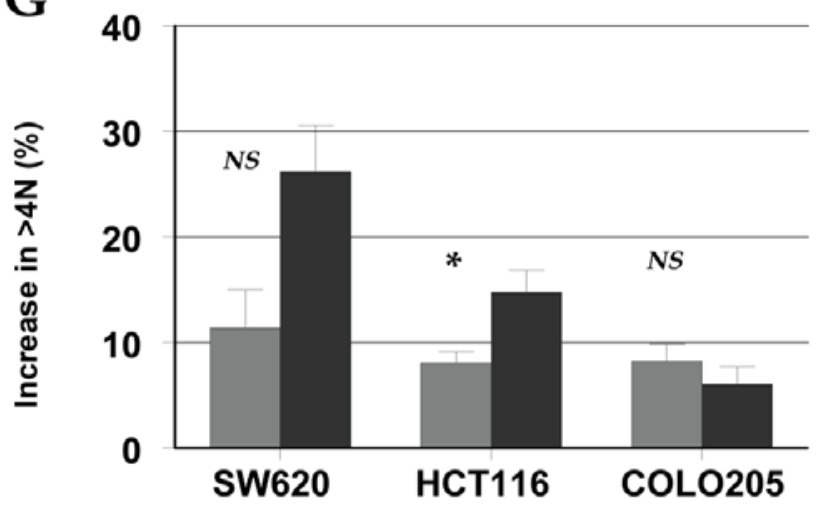

Minipump experiments

Control Barasertib

Dosed at $25 \mathrm{mg} / \mathrm{kg}$ (i.p) and $150 \mathrm{mg} / \mathrm{kg} /$ day (m.p) $P<0.05=* \quad P<0.01=\dagger P<0.001=\ddagger$

Figure 2. Antitumor and PD effects of barasertib in colorectal tumor xenograft models. Female nude mice bearing established (A) SW620, (B) HCT116, (C) Colo205 and (D) SW620 human CRC tumor xenografts were constantly infused with a s.c. minipump over $48 \mathrm{~h}$ with either drug vehicle (n=12) or barasertib $(\mathrm{n}=8,150 \mathrm{mg} / \mathrm{kg} / \mathrm{day})(\mathrm{A}-\mathrm{C})$; or i.p. dosed once a day for 4 days (Q1Dx4 i.p.) with either drug vehicle ( $\mathrm{n}=12)$ or barasertib (n=10, $25 \mathrm{mg} / \mathrm{kg} / \mathrm{day})(\mathrm{D})$. Satellite groups of animals $(n=3)$ were sacrificed at the end of minipump infusion and assessed for phH3 expression (E) and DNA content; either 4N (G2/M phase) $(\mathrm{F})$ and $>4 \mathrm{~N}$ (ploidy) $(\mathrm{G})$. All xenograft data points represent mean tumor volume $\left(\mathrm{cm}^{3} \pm \mathrm{SEM}\right)$. Flow cytometry data represent the group average $( \pm \mathrm{SEM})$. Statistical analysis was carried out using standard 1 tailed Student's t-test; ns, not significant; ${ }^{*} \mathrm{p}<0.05,{ }^{\dagger} \mathrm{p}<0.01,{ }^{\dagger} \mathrm{p}<0.001$. 
Table II. Macroadenoma analysis after a 3-week treatment cycle of barasertib in $A p c^{\mathrm{Min} /+}$ mice.

\begin{tabular}{lrrr}
\hline & \multicolumn{3}{c}{ Adenoma survey } \\
\cline { 2 - 4 } & Control & $\begin{array}{c}\text { Barasertib } \\
(25 \mathrm{mg} / \mathrm{kg} / \text { day })\end{array}$ & $\begin{array}{c}\text { Student's t-test } \\
\text { P-value }\end{array}$ \\
\hline No. of adenomas (SB All) & & $31.87 \pm 5.64$ & 0.02 \\
No. of adenomas (Colon) & $52.31 \pm 6.18$ & $1.20 \pm 0.30$ & $\mathrm{NS}$ \\
Diameter of adenomas (mm) (SB All) & $1.92 \pm 0.33$ & $0.78 \pm 0.08$ & $\mathrm{NS}$ \\
Diameter of adenomas (mm) (Colon) & $0.94 \pm 0.02$ & $0.79 \pm 0.22$ & $\mathrm{NS}$ \\
Tumor burden $\left(\mathrm{mm}^{3}\right.$ ) (SB All) & $1.14 \pm 0.20$ & $12.67 \pm 2.13$ & 0.02 \\
Tumor burden $\left(\mathrm{mm}^{3}\right.$ ) (Colon) & $22.09 \pm 3.14$ & $3.49 \pm 1.72$ & $\mathrm{NS}$
\end{tabular}

Macroscopic lesions were surveyed across the gastrointestinal track of $A p c^{\mathrm{Min} /+}$ mice after 3 weeks of treatment with either control (n=13) vehicle or barasertib $(n=15)$. Data were summarized using the mean \pm SEM.

Table III. Effects of barasertib on microscopic adenoma burden in $A p c^{\mathrm{Min} /+}$ mice.

\begin{tabular}{lccccc}
\hline & & \multicolumn{3}{c}{ Microadenoma no. } \\
\cline { 3 - 6 } $\begin{array}{l}\text { Gut } \\
\text { segment }\end{array}$ & $\begin{array}{c}\text { Adenoma } \\
\text { category }\end{array}$ & Control & $\begin{array}{c}\text { Barasertib } \\
(25 \mathrm{mg} / \mathrm{kg} / \mathrm{day})\end{array}$ & $\begin{array}{c}\text { Student's t-test } \\
\text { P-value }\end{array}$ & $\begin{array}{c}\text { Percentage } \\
\text { reduction }(\%)\end{array}$ \\
\hline Small & 1 & $20.0 \pm 4.5$ & $17.0 \pm 3.7$ & $\mathrm{NS}$ & 15 \\
bowel & 2 & $35.2 \pm 6.4$ & $12.8 \pm 3.5$ & 0.01 & 6.01 \\
& 3 & $19.5 \pm 4.2$ & $6.3 \pm 1.7$ & 0.04 & 68 \\
\hline
\end{tabular}

Microscopic adenomas were counted across the distal segment of the small bowel, visualized under H\&E staining, measurements performed with a stage calibrated graticule. Data were summarized using the mean \pm SEM.

Effect of barasertib on pharmacodynamic endpoints and cell proliferation in vivo. Aurora-B phosphorylates histone $\mathrm{H} 3$ at Ser10, exclusively during mitosis in mammalian cells (28). Samples for pharmacodynamic endpoints were collected $2 \mathrm{~h}$ post the last dose of barasertib (i.p. $A p c^{\mathrm{Min} /+}$ ). Absent IHC staining of phH3 (Ser10) was taken as direct evidence of inhibition of ABK activity (Fig. 3).

In the adenomatous tissue, the number and proportion (\%) of phH3-positive cells in the barasertib versus the vehicletreated group were markedly reduced by $97 \%(\mathrm{P}<0.001)$ and 94\% ( $\mathrm{P}<0.001)$, respectively; Table IV, Fig. 3. Adenomas were $53 \%$ smaller on average $(\mathrm{P}<0.001$, Table IV) in the barasertib versus vehicle-treated group and the adenomas displayed a noticeable trend of a greater number of apoptotic bodies when examined after H\&E staining. Further, a similar trend was observed following cleaved caspase-3 staining (data not shown). BrdU uptake in the adenomas experienced a decrease of $51 \%(\mathrm{P}=0.01$, Table IV), indicating a possible lower division rate in the adenoma. The comparison of BrdU labeling indices across treated and control group did not reveal much difference in BrdU uptake, but this could be explained by the smaller overall size of barasertib-treated adenomas (Table IV). Normal non-adenomatous tissue displayed similar effects but with no major changes in tissue renewal (data not shown).

\section{Discussion}

Previous research has shown that Aurora kinase levels are elevated in several cancer types, including CRC and that the Aurora kinase family may represent potential targets for therapeutic intervention (29). Studies in in vitro and in vivo tumor models support this view, and have shown that inhibiting ABK activity produces significant antitumor effects in both hematological (30) and solid tumors including hepatocellular cancer (31). Recent work in CRC has pointed towards a role for mutant APC in activating ABK, via survivin; leading to increased mitotic rates and a mechanism by which colon tumorigenesis is promoted (12). Here we investigate further the potential of targeting GI tract cancers using the selective ABK prodrug, barasertib. Barasertib-hQPA (the active moiety of barasertib) inhibits tumor cell growth and induces apoptosis in a concentration- and time-dependent manner across a panel of CRC cell lines. Consistent with previous reports $(32,33)$, barasertib-hQPA caused a lower induction in apoptosis in HCT116 cells and required prolonged exposures (Fig. 1F). A similar effect was observed in SW620 cells whereas in Colo205 and RKO cells the extent of apoptosis was more pronounced, reaching $40-60 \%$ at concentrations of $0.1 \mu \mathrm{mol} / 1$ barasertib-hQPA. The fact that cell growth was inhibited after 

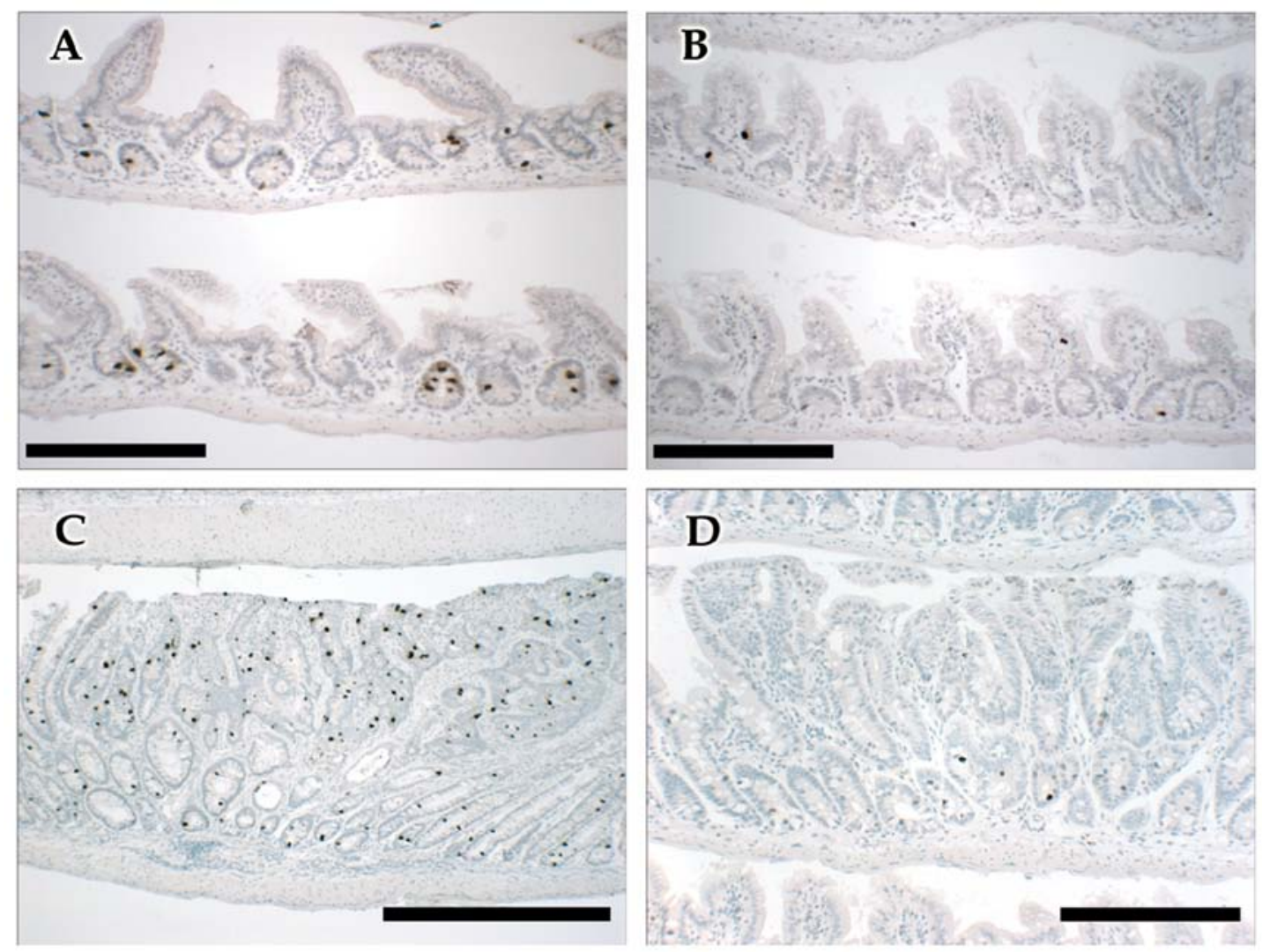

Figure 3. Barasertib pharmacodynamic effects on phH3 levels in small intestine of $\mathrm{Apc}^{\mathrm{Min} /}$ mice. Normal tissue (A and B) and adenomas (C and D) from small intestines following 3 weeks of treatment with vehicle (A and C) or barasertib (B and D), stained for phH3 expression by IHC. Scale bars, $200 \mu \mathrm{m}$ (A, B and D) and $500 \mu \mathrm{m}(\mathrm{C})$.

Table IV. The effects of barasertib treatment on proliferation markers in adenomas of the small bowel of $A p c^{\mathrm{Min} /+}$.

\begin{tabular}{lccc}
\hline & \multicolumn{3}{c}{ Proliferation assessment } \\
\cline { 2 - 4 } & Control & $\begin{array}{c}\text { Barasertib } \\
(25 \mathrm{mg} / \mathrm{kg} / \mathrm{day})\end{array}$ & $\begin{array}{c}\text { Student's t-test } \\
\text { P-value }\end{array}$ \\
\hline BrdU $^{+}$per adenoma & $241.23 \pm 36.05$ & $117.01 \pm 31.66$ & 0.015 \\
Mitoses per adenoma & $7.24 \pm 1.10$ & $5.98 \pm 1.60$ & $\mathrm{NS}$ \\
phH3 & $1.00 \pm 0.34$ & $<0.001$ \\
Cells per adenoma & $37.76 \pm 5.19$ & $537.17 \pm 67.67$ & 0.001 \\
BrdU labeling index $(\%)$ & $1143.44 \pm 156.75$ & $21.78 \pm 1.34$ & $\mathrm{NS}$ \\
Mitotic index (\%) & $21.10 \pm 1.18$ & $1.11 \pm 0.27$ & $\mathrm{NS}$ \\
phH3 labeling index (\%) & $0.63 \pm 0.10$ & $0.19 \pm 0.09$ & $<0.001$ \\
\hline
\end{tabular}

Proliferation in the adenomas was analysed by immunohistochemistry staining for BrdU incorporation, phH3 and mitoses present in the tissue of interest of barasertib and vehicle treated mice. Data were summarized using the mean \pm SEM.

a $24 \mathrm{~h}$ exposure to barasertib-hQPA followed by extended washout periods suggests that an intermittent schedule may be an effective treatment in CRC. The difference in response rates to barasertib between the CRC cell lines may underlie differences in the expression of proteins involved in cell cycle checkpoint/apoptotic pathways as well as differences in growth rate, $\%$ of polyploidy and /or DNA content of polyploidy cells. Differences in intracellular drug uptake between cell lines could also be responsible; previous studies on the effects of barasertib in AML cells have indicated expression of the transporter proteins P-glycoprotein and BCRP1 can have a negative impact on barasertib-hQPA efficacy (34).

We also demonstrated that short-term treatment $(48 \mathrm{~h}$, osmotic minipump) with barasertib produced durable antitumor effects in vivo in three established subcutaneous CRC xenografts in nude mice. Similar preclinical activity has recently 
been documented in both a CRC cell line-derived xenograft (HT29) and 3 patient-derived explants with ENMDA-2076, a multi-targeted anti-angiogenic and Aurora kinase inhibitor (35). Whilst Colo205 cells more readily underwent apoptosis following barasertib treatment in vitro and also appeared to be the most sensitive model in vivo, barasertib induced significant antitumor activity in both SW620 and HCT116 models. Differences between in vitro and in vivo barasertib data may reflect the multi-cellular contribution of the tumor microenvironment (including stroma) found in tumor xenografts versus the mono-cellular conditions of cell culture assays used in this study. Pharmacodynamic analysis in CRC xenografts indicated the antitumor effects were associated with reduced phH3 expression and accumulation of cells with $4 \mathrm{~N}$ and $>4 \mathrm{~N}$ DNA content. These findings are consistent with failed chromosome separation and thus failed cytokinesis but continued cell cycle progression following inhibition of ABK activity. These data indicate that the mechanism of action of barasertib in vivo recapitulates that observed with barasertib-hQPA in vitro and with other ABK inhibitors $(13,36)$. Interestingly, pharmacodynamic analysis of barasertib-treated Colo205 xenografts did not indicate such a strong trend for enriched $4 \mathrm{~N}$ and ploidy $(>4 \mathrm{~N})$ tumor cells. Colo205 cells were observed to be very pro-apoptotic in in vitro assays and sensitive in in vivo growth inhibition studies. The weaker trend in increased DNA content following barasertib treatment in vivo may have reflected cells undergoing early apoptosis rather than endo-reduplication. Consistent with this, flow cytometric analysis of tumor cells from Colo205 xenografts showed there to be a higher proportion with a sub-G1 DNA content, a marker of apoptosis in this model (data not shown).

Inhibition of ABK activity in an autochthonous model of CRC, such as the $A p c^{\mathrm{Min} /+}$ mouse which is considered as well validated model of intestinal cancer, has not yet been reported (37,38). The $A p c^{\mathrm{Min} /+}$ mouse has several advantages over standard CRC in vivo models, such as human tumor xenografts, in that the mouse strain is immunocompetent and the adenomas grow in situ within the context of the complex intestinal mucosal microenvironment. In the $A p c^{\mathrm{Min} /+}$ mouse model we found a marked reduction in the number of detectable adenomas throughout the SB following the three-week treatment with barasertib, with the most pronounced effect being seen in the distal segment where most of the lesions occurred. In addition to the reduction in the number of adenomas, the sizes of the lesions in the SB were also reduced leading to a significant reduction in tumor burden. Tumor burden in the colon was also reduced, but this did not reach statistical significance and is most likely due to the relatively small number of adenomas that are found in the colon. The number of colonic adenomas was low in the current study, but it is known that the number of colonic adenomas in the $A p c^{\mathrm{Min} /+}$ mouse is variable, and may be in part be a reflection the inflammatory environment in the intestines which can be affected by gut micro-flora which in turn can be influenced by specific pathogen-free conditions of a vivarium $(39,40)$.

Microscopic analysis refined the quantification of adenomas and confirmed that the $A p c^{\mathrm{Min} /+}$ model developed adenomas of various sizes, and demonstrated that the incidence of all but the smallest adenomas (those occupying space within a single villus) were substantially reduced by treatment with barasertib. It is important to note that under treatment larger adenomas were less numerous (category 3 and 4) potentially due to the pro-apoptotic and anti-proliferative effects of barasertib. It is also possible that barasertib treatment could de-bulk larger adenomas to smaller sizes (e.g. from category 3 or 4 to category 1), and therefore account for the reason why the difference in category 1 adenomas between barasertib and vehicle control $A p c^{\mathrm{Min} /+}$ mice was not statistically significant in this study. From these data we cannot fully ascertain whether the effects of barasertib are to prevent adenoma growth, to shrink adenomas, or both. Further work (including short-term exposures) would be required to investigate this further.

Scoring the histological sections of SB from barasertib treated $A p c^{\mathrm{Min} /+}$ mice revealed a substantial suppression of ABK activity in both histologically normal crypts and adenomas, as measured by phH3 immunostaining. Mitotic index was also increased in both normal crypts and adenomas. Taken together, the in vivo data indicate that the biological response of intestinal tissue in $A p c^{\mathrm{Min} /+}$ mice parallels the response of SW620 and HCT116 xenografts following inhibition of ABK activity by barasertib. Although not directly comparable, it is noteworthy that the extent of reduced phH3 staining following barasertib in the SW620 xenograft by flow cytometry appeared substantially less than that seen in the adenomas/crypts from $A p c^{\mathrm{Min} /+}$ mice. However, this may have reflected differences in the methodologies used and the duration of barasertib treatment which was much shorter in the CRC xenografts. Furthermore, the tumor burden was much larger in the CRC xenograft studies compared with the $A p c^{\mathrm{Min} /+}$ mouse study, which could in part contribute to a smaller effect on phH3 levels. Recent work by Zhang et al address the relationship of APC regulating ABK activity and its effect of phH3 distribution in colonic crypt via survivin, an increase in ABK activity leads to higher phH3 position within APC mutated crypts and adenoma (12). Interestingly, the IHC phH3 staining pattern in the vehicle control sections of the $A p c^{\mathrm{Min} /+}$ mice (Fig. 3) was reminiscent of the clinical counterparts described the Zhang et al study (12). Namely, phH3 staining confined to lower intestinal crypts in the normal tissue and a more stochastic distribution throughout the adenomas.

The lack of an apparent effect of barasertib on BrdU labeling index is likely to have resulted from smaller adenomas and the rapid apoptosis of cells undergoing aberrant mitosis. Since apoptotic cells are likely to be promptly eliminated from the adenoma tissue (and therefore not contribute significantly to the assessment of labeling index), the overall proportion of cells in S-phase could appear relatively unaffected, in particular if the tumor was shrinking in response to drug treatment. Although we did not directly assess tumor shrinkage in the present study, the number of cells per adenoma was significantly lower in barasertib-treated animals compared with controls. Assessment of cleaved caspase- 3 showed a trend in apoptosis accumulation following treatment although this effect was not significant (data not shown). The later may be explained due to the nature of the intestinal gut, being a site of high cell turnover, which would have allowed for the clearing of apoptotic cells prior to analysis. Future work taking earlier samples for analysis may give additional insights into these results. 
Our data in CRC cell lines and xenografts and in $A p c^{\mathrm{Min}+}$ mice provide supportive evidence that barasertib treatment inhibits $\mathrm{ABK}$ and reduces phH3 levels in dividing tumor cells. This results in aberrant mitosis, aneuploidy, polyploidy and apoptosis thereby suppressing the growth of tumor xenografts or intestinal adenomas. The results of the present study confirm that ABK activity plays a substantial role in the growth of established CRC tumor models, but more interestingly it also plays a role in the growth of very early pre-carcinogenic lesions in the intestinal tract of $\mathrm{Apc}^{\mathrm{Mint}}$ mice. Selective ABK inhibitors are currently being evaluated in various clinical trials and the present study provides a rationale for extending the scope of clinical evaluation to include earlier stages of CRC. Barasertib has been tested in both solid and hematological cancer patients. In the case of solid tumors a 2-h infusion schedule, every 7 or 14 days, was investigated (41). Neutropenia was the most frequently reported adverse event/ dose-limiting toxicity and the best observed responses in this trial were prolonged disease stabilizations in some patients. As with other therapies targeted against cycling cells, care and attention will be needed to identify the optimum dosing regime in order to achieve a good therapeutic margin.

\section{Acknowledgements}

We thank Dr Kate Byth at AstraZeneca for helpful feedback and discussions during the drafting of this manuscript. We acknowledge the assistance of both the CDMG (AstraZeneca) and Clare Hall (CRUK) for in vivo efficacy studies. Further, we thank George Elia for immunohistochemistry support. Financial support for this study was provided by AstraZeneca. D.A. is funded by a Biology and Biotechnology Science Research Council (BBSRC) CASE research studentship in conjunction with AstraZeneca.

\section{References}

1. Jemal A, Siegel R, Ward E, Hao Y, Xu J and Thun MJ: Cancer statistics, 2009. CA Cancer J Clin 59: 225-249, 2009.

2. Chee CE and Sinicrope FA: Targeted therapeutic agents for colorectal cancer. Gastroenterol Clin North Am 39: 601-613, 2010.

3. Keen $\mathrm{N}$ and Taylor $\mathrm{S}$ : Aurora-kinase inhibitors as anticancer agents. Nat Rev Cancer 4: 927-936, 2004.

4. Yang KT, Li SK, Chang CC, et al: Aurora-C kinase deficiency causes cytokinesis failure in meiosis I and production of large polyploid oocytes in mice. Mol Biol Cell 21: 2371-2383, 2010.

5. Bischoff JR, Anderson L, Zhu Y, et al: A homologue of Drosophila Aurora kinase is oncogenic and amplified in human colorectal cancers. EMBO J 17: 3052-3065, 1998.

6. Pohl A, Azuma M, Zhang W, et al: Pharmacogenetic profiling of Aurora kinase B is associated with overall survival in metastatic colorectal cancer. Pharmacogenomics J 11: 93-99, 2011.

7. Fearon ER and Vogelstein B: A genetic model for colorectal tumorigenesis. Cell 61: 759-767, 1990.

8. Ilyas M, Straub J, Tomlinson IP and Bodmer WF: Genetic pathways in colorectal and other cancers. Eur J Cancer 35: 1986-2002, 1999.

9. Senda T, Iizuka-Kogo A, Onouchi $\mathrm{T}$ and Shimomura A: Adenomatous polyposis coli (APC) plays multiple roles in the intestinal and colorectal epithelia. Med Mol Morphol 40: 68-81, 2007.

10. Hanson CA and Miller JR: Non-traditional roles for the Adenomatous Polyposis Coli (APC) tumor suppressor protein. Gene 361: 1-12, 2005.

11. Boman BM, Walters R, Fields JZ, et al: Colonic crypt changes during adenoma development in familial adenomatous polyposis: immunohistochemical evidence for expansion of the crypt base cell population. Am J Pathol 165: 1489-1498, 2004.
12. Zhang T, Fields JZ, Opdenaker L, et al: Survivin-induced Aurora-B kinase activation: A mechanism by which APC mutations contribute to increased mitoses during colon cancer development. Am J Pathol 177: 2816-2826, 2010.

13. Wilkinson RW, Odedra R, Heaton SP, et al: AZD1152, a selective inhibitor of Aurora B kinase, inhibits human tumor xenograft growth by inducing apoptosis. Clin Cancer Res 13: 3682-3688, 2007.

14. Preston S, Leedham S, Oukrif D, et al: The development of duodenal microadenomas in FAP patients: the human correlate of the Min mouse. J Pathol 214: 294-301, 2008.

15. Minde DP, Anvarian Z, Rüdiger SG and Maurice MM: Messing up disorder: how do missense mutations in the tumor suppressor protein APC lead to cancer? Mol Cancer 10: 101, 2011.

16. Chen LC, Hao CY, Chiu YS, et al: Alteration of gene expression in normal-appearing colon mucosa of APC(min) mice and human cancer patients. Cancer Res 64: 3694-3700, 2004.

17. Corpet DE and Pierre F: How good are rodent models of carcinogenesis in predicting efficacy in humans? A systematic review and meta-analysis of colon chemoprevention in rats, mice and men. Eur J Cancer 41: 1911-1922, 2005.

18. Mortlock AA, Foote KM, Heron NM, et al: Discovery, synthesis, and in vivo activity of a new class of pyrazoloquinazolines as selective inhibitors of aurora B kinase. J Med Chem 50: 2213-2224, 2007

19. Cummings J, Hodgkinson C, Odedra R, et al: Preclinical evaluation of M30 and M65 ELISAs as biomarkers of drug induced tumor cell death and antitumor activity. Mol Cancer Ther 7: 455-463, 2008

20. Wedge SR, Ogilvie DJ, Dukes M, et al: ZD4190: an orally active inhibitor of vascular endothelial growth factor signaling with broad-spectrum antitumor efficacy. Cancer Res 60: 970-975, 2000.

21. Widrow RJ, Rabinovitch PS, Cho K and Laird CD: Separation of cells at different times within G2 and mitosis by cyclin B1 flow cytometry. Cytometry 27: 250-254, 1997.

22. Alferez D, Ryan A, Goodlad R, Wright N and Wilkinson R: Effects of vandetanib on adenoma formation in a dextran sodium sulphate enhanced $\mathrm{Apc}^{\mathrm{MIN} /+}$ mouse model. Int J Oncol 37: 767-772, 2010.

23. Alferez D, Wilkinson RW, Watkins J, et al: Dual inhibition of VEGFR and EGFR signaling reduces the incidence and size of intestinal adenomas in Apc(Min/+) mice. Mol Cancer Ther 7: 590-598, 2008

24. Goodlad RA, Ryan AJ, Wedge SR, et al: Inhibiting vascular endothelial growth factor receptor-2 signaling reduces tumor burden in the ApcMin/+ mouse model of early intestinal cancer. Carcinogenesis 27: 2133-2139, 2006.

25. Alferez D and Goodlad R: To best measure cell proliferation in samples from the intestine. Cell Prolif 40: 231-240, 2007.

26. Nair JS, Ho AL, Tse AN, et al: Aurora B kinase regulates the postmitotic endoreduplication checkpoint via phosphorylation of the retinoblastoma protein at serine 780. Mol Biol Cell 20: 2218-2228, 2009.

27. Ditchfield C, Johnson VL, Tighe A, et al: Aurora B couples chromosome alignment with anaphase by targeting BubR1, Mad2, and Cenp-E to kinetochores. J Cell Biol 161: 267-280, 2003.

28. Ota T, Suto S, Katayama H, et al: Increased mitotic phosphorylation of histone $\mathrm{H} 3$ attributable to AIM-1/Aurora-B overexpression contributes to chromosome number instability. Cancer Res 62: 5168-5177, 2002.

29. Lens SM, Voest EE and Medema RH: Shared and separate functions of polo-like kinases and aurora kinases in cancer. Nat Rev Cancer 10: 825-841, 2010.

30. Oke A, Pearce D, Wilkinson RW, et al: AZD1152 rapidly and negatively affects the growth and survival of human acute myeloid leukemia cells in vitro and in vivo. Cancer Res 69: 4150-4158, 2009.

31. Aihara A, Tanaka S, Yasen M, et al: The selective Aurora B kinase inhibitor AZD1152 as a novel treatment for hepatocellular carcinoma. J Hepatol 52: 63-71, 2010.

32. Nair JS, de Stanchina E and Schwartz GK: The topoisomerase I poison CPT-11 enhances the effect of the aurora B kinase inhibitor AZD1152 both in vitro and in vivo. Clin Cancer Res 15: 2022-2030, 2009.

33. Azzariti A, Bocci G, Porcelli L, et al: Aurora B kinase inhibitor AZD1152: determinants of action and ability to enhance chemotherapeutics effectiveness in pancreatic and colon cancer. Br J Cancer 104: 769-780, 2011. 
34. Grundy M,Seedhouse C, Russell NH and Pallis M: P-glycoprotein and breast cancer resistance protein in acute myeloid leukaemia cells treated with the aurora-B kinase inhibitor barasertib-hQPA. BMC Cancer 11: 254, 2011.

35. Tentler JJ, Bradshaw-Pierce EL, Serkova NJ, et al: Assessment of the in vivo antitumor effects of ENMD-2076, a novel multitargeted kinase inhibitor, against primary and cell line-derived human colorectal cancer xenograft models. Clin Cancer Res 16 : 2989-2998, 2010.

36. Girdler F, Gascoigne KE, Eyers PA, et al: Validating Aurora $\mathrm{B}$ as an anti-cancer drug target. J Cell Sci 119: 3664-3675, 2006.

37. Boivin GP, Washington $\mathrm{K}$, Yang K, et al: Pathology of mouse models of intestinal cancer: consensus report and recommendations. Gastroenterology 124: 762-777, 2003.
38. Paulsen JE: Modulation by dietary factors in murine FAP models. Toxicol Lett 112-113: 403-409, 2000.

39. Rhodes JM and Campbell BJ: Inflammation and colorectal cancer: IBD-associated and sporadic cancer compared. Trends Mol Med 8: 10-16, 2002.

40. Alison M: The Cancer Handbook. John Wiley \& Sons, Chichester, West Sussex, Hoboken, NJ, 2007.

41. Boss DS, Witteveen PO, van der Sar J, et al: Clinical evaluation of AZD1152, an i.v. inhibitor of Aurora B kinase, in patients with solid malignant tumors. Ann Oncol 22: 431-437, 2011. 